\title{
Light Control of Localized Photobioconvection
}

\author{
Jorge Arrieta ${ }^{1,},{ }^{1 *}$ Marco Polin, ${ }^{2, \dagger}$ Ramón Saleta-Piersanti, ${ }^{1}$ and Idan Tuval ${ }^{1, \$}$ \\ ${ }^{1}$ Instituto Mediterráneo de Estudios Avanzados, IMEDEA, UIB-CSIC, Esporles, 07190, Spain \\ ${ }^{2}$ Physics Department and Centre for Mechanochemical Cell Biology, University of Warwick, \\ Gibbet Hill Road, Coventry, CV4 7AL, United Kingdom
}

(Received 4 February 2019; revised manuscript received 4 July 2019; published 11 October 2019)

\begin{abstract}
Microorganismal motility is often characterized by complex responses to environmental physicochemical stimuli. Although the biological basis of these responses is often not well understood, their exploitation already promises novel avenues to directly control the motion of living active matter at both the individual and collective level. Here we leverage the phototactic ability of the model microalga Chlamydomonas reinhardtii to precisely control the timing and position of localized cell photoaccumulation, leading to the controlled development of isolated bioconvective plumes. This novel form of photobioconvection allows a precise, fast, and reconfigurable control of the spatiotemporal dynamics of the instability and the ensuing global recirculation, which can be activated and stopped in real time. A simple continuum model accounts for the phototactic response of the suspension and demonstrates how the spatiotemporal dynamics of the illumination field can be used as a simple external switch to produce efficient bio mixing.
\end{abstract}

DOI: 10.1103/PhysRevLett.123.158101

The autonomous movement of microorganisms has fascinated scientists since the discovery of the microbial world. Particularly striking is the variety of coordinated collective dynamics that emerges with startling reliability in groups of motile microorganisms, from traffic lanes and oscillations in bacterial swarms [1,2], to wolf-pack hunting [3] and microbial morphogenesis [4,5]. Nowadays, microbial motility is an important part of a growing interdisciplinary field aiming to uncover the fundamental laws governing the dynamics of so-called active matter [6], eventually allowing us to harness micron-scale motility for applications ranging from targeted payload delivery [7] to direct assembly of materials [8,9], with either living organisms or synthetic microswimmers [10-12]. Realizing this potential will hinge on our ability to alter and ultimately control the motion of both individual cells and microbial collectives.

Microbial motility can be controlled through clever engineering of boundaries [13-15] or topology [16,17] of the vessels holding the microbial suspension, leading for example to predictable accumulation [18-20] or circulation of cells [14,21-23]. However, together with strategies squarely rooted in physics, control of living microswimmers can rely also on approaches bridging between physics and biology, by taking advantage of pathways linking motility with the perception of physicochemical stimuli by cells. Light is particularly well suited to this end: its manipulation is readily achievable at both the macroscopic and microscopic [24,25] scales; it is an important stimulus for a wide variety of microorganisms, providing both energy [26] and information often used to prevent potentially lethal light-induced stress [27]. Most microorganisms respond to light by linking swimming speed to light intensity (photokinesis, [28]) and/or redirecting their motion towards or away from the light source (phototaxis, [29-31]). Although the physiological details underpinning these active responses are often not completely understood [30-33], techniques employing light to precisely control the dynamics of swimming microorganisms are already emerging. Biological responses to light led to the development of genetically engineered light-sensitive bacteria [34-36] used, for example, to power micron-sized motors [23], and have even inspired the fabrication of light-reactive artificial swimmers [11,37-39].

Within eukaryotes together with recent studies on Euglena gracilis (Protozoa) [40-43], current applications focus in particular on the model unicellular green alga Chlamydomonas reinhardtii (CR), and range from microcargo delivery by individual cells [44] to trapping of passive colloids by light-induced hydrodynamic tweezers [45], and photofocusing of algal suspensions through an interplay of photo and gyro taxis [46]. Unlocking the full potential of light-based control, however, will require the development of techniques based on a collective response that is both quick and localized. Despite considerable progress, this is not currently available.

Here we exploit the phototactic response of $\mathrm{CR}$ to demonstrate a novel form of dynamic control of a cell suspension, based on a fast ( $10 \mathrm{~s})$ accumulation that can be localized anywhere within the suspension. Cells photoaccumulate around the light from a horizontal optical fiber [Fig. 1(b)] and act as a miniaturized pump driving a global 


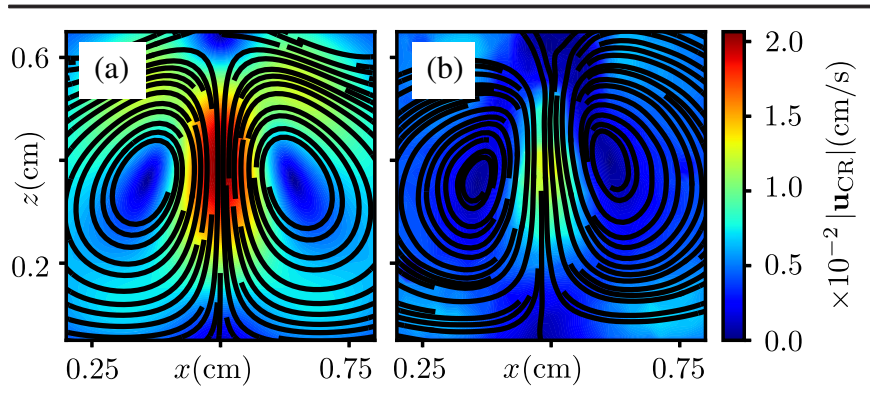

FIG. 1. Contours of the cells averaged velocity field obtained from (a) the numerical integration of (1)-(3) for $n_{0}=1.5 \times$ $10^{7}$ cells $/ \mathrm{ml}$ and $\beta=0.14$ and (b) from the PIV analysis of experimental data. Solid black lines represent in both panels the corresponding streamlines.

recirculation of the suspension with a fast response time, quantitatively captured by a simple model [Fig. 1(a)]. The fast response of the suspension can be exploited for efficient biomixing, an attractive solution to improve current photobioreactor technology for biofuel production where mixing is essential to distribute nutrients, and transfer gases across gas-liquid interfaces [47-49]. Our results serve as a proof of principle for more complex instances of light-controlled fluid flows in biological suspensions.

Unicellular biflagellate green algae Chlamydomonas reinhardtii wild type strain $\mathrm{CC} 125$ were grown axenically at $20^{\circ} \mathrm{C}$ in Tris-Acetate-Phosphate medium (TAP) [50] under fluorescent light illumination (OSRAM Fluora, $100 \mu \mathrm{mol} /\left(\mathrm{m}^{2} \mathrm{~s}\right)$ PAR) following a $14 \mathrm{~h}(10 \mathrm{~h})$ light (dark) diurnal cycle. Exponentially growing cells were harvested, photoaccumulated, diluted to the target concentration with fresh TAP, and loaded in a vertical observation chamber formed by a square shaped Agar-TAP gasket of $L=1 \mathrm{~cm}$ side and $h=1 \mathrm{~mm}$ thickness, sandwiched between two coverslips. The main experiments were performed at two average concentrations: $n_{0}^{\mathrm{h}}=1.5 \times 10^{7}$ cells $/ \mathrm{ml}$ (eight repeats); and $n_{0}^{1}=7 \times 10^{6}$ cells $/ \mathrm{ml}$ (six repeats). Tests for plume formation were also conducted at $n_{0}=6.1$, $9.0 \times 10^{6}, 1.24,1.40 \times 10^{7}$ cells $/ \mathrm{ml}$ The suspension's dynamics was visualized through darkfield illumination at $635 \mathrm{~nm}$ (FLDR-i70A-R24, Falcon Lighting Germany) and recorded by a CCD camera (Pike, AVT USA) hosted on a continuously focusable objective (InfiniVar CFM-2S, Infinity USA). Localized actinic illumination was provided by a $200 \mu \mathrm{m}$ diameter horizontal multimode optical fiber (FT200EMT, Thorlabs USA) coupled to a $470 \mathrm{~nm}$ highpower LED (M470L2, Thorlabs USA). The fiber's output intensity $I(\mathbf{x})$, centered at $\mathbf{x}_{\mathrm{fb}}=\left(x_{\mathrm{fb}}, z_{\mathrm{fb}}\right)$ [Fig. 2(a)], is well approximated by the Gaussian used in numerical simulations throughout the manuscript (width $\sigma_{I}=667 \mu \mathrm{m}$; peak intensity $I_{0}=260 \mu \mathrm{mol} /\left(\mathrm{m}^{2} \mathrm{~s}\right)$; Fig. S1[51]).

Figure 2 shows the evolution of the photoaccumulation dynamics for $n_{0}^{\mathrm{h}}$. Without light stimuli, individual cells swim in a characteristic run-and-tumble-like behavior [52] leading to a uniform spatial distribution at the population level. As the actinic light is switched on, phototactic cells start accumulating around the fiber, through a characteristic phototactic steering mechanism [33] based on an interplay between time-dependent stimulation of a light-sensitive organelle [53,54] and the ensuing flagellar response [55]. Phototaxis leads, within $\sim 10 \mathrm{~s}$, to a $\sim 2$-mm-wide region of
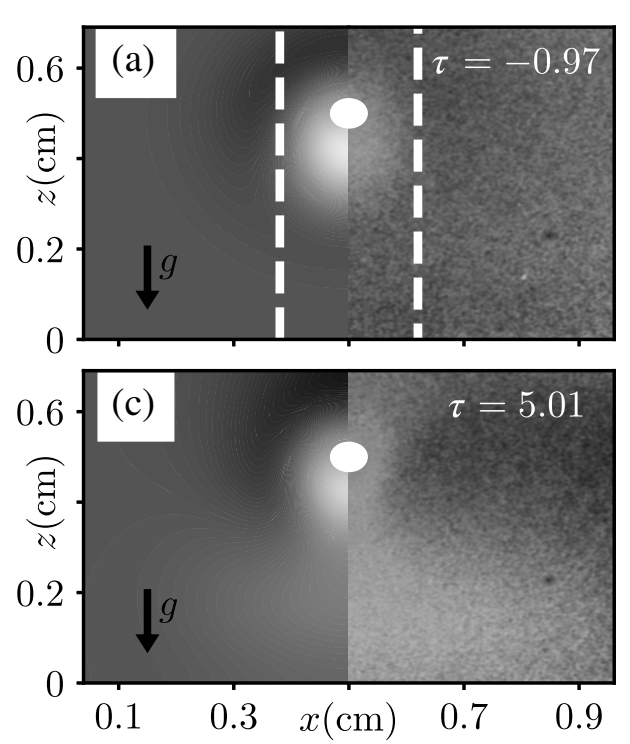
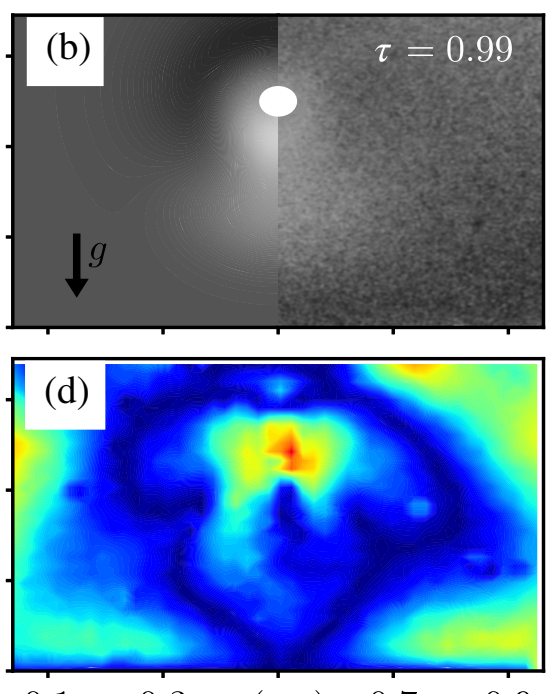

0.1
0.3 $x(\mathrm{~cm})$
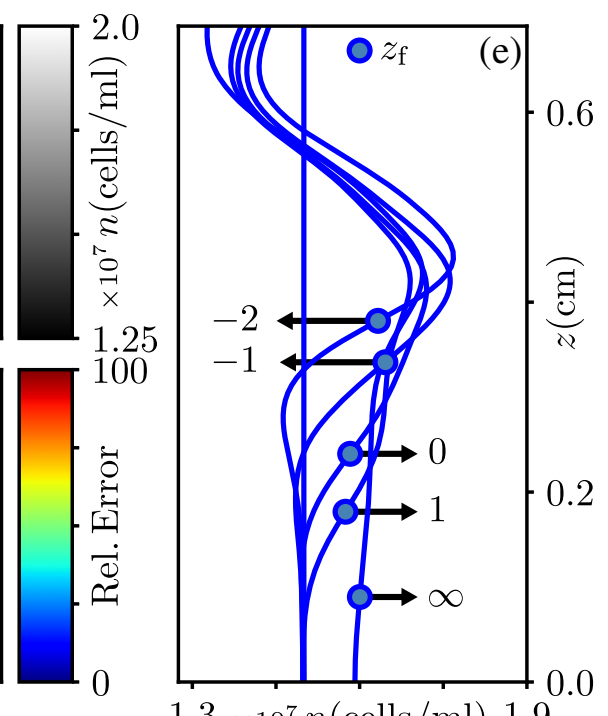

$1.3 \times 10^{7} n($ cells $/ \mathrm{ml}) 1.9$

FIG. 2. Dynamics of plume formation. (a)-(c) Evolution of photoaccumulation and plume formation at different values of the normalized time $\tau$. Panels show the cell density from the continuum model, $n$ (left side), and the dark-field image from the corresponding experiment at the same value of $\tau$. White dots in (a)-(c) correspond with the position of the light fiber. (d) Relative error between average experimental and numerical flows of cells (each flow field has been first rescaled by its maximum value). (e) Average vertical cell concentration profiles for the specified values of $\tau$, and corresponding values of $z_{\mathrm{f}}$ (circles). 
high cell concentration [31]. This is gravitationally unstable, and eventually falls forming a single, localized sinking plume of effectively denser fluid [Figs. 2(b) and 2(c) and the Supplemental Material, Movie S1 [51]]. The system converges to its steady state as the plume reaches the bottom of the container ( $\sim 30 \mathrm{~s})$, two orders of magnitude faster than reported for alternative configurations [45] with the cells advected along the strong global recirculation seen in Fig. 1(b) (experimental flow of cells obtained with OpenPIV using cells as tracers [56]). This buoyancy-driven instability is reminiscent of bioconvection, one of the best known collective phenomena in suspensions of microswimmers [57-59]. Here it can be understood as a lightinduced instance of a single bioconvective plume, which can be actively modulated by light and localized anywhere within the sample. Cell accumulation, however, does not always lead to plumes. In samples with average concentration $n_{0}^{1}$, the photoaccumulated high-concentration region does not sink to the bottom but reaches instead a stable height just below the fiber's center. Despite the absence of a proper plume, however, the background fluid is still globally stirred (see the Supplemental Material, Movie S2 [51]).

Sinking of the initial region of photoaccumulated cells can be quantified from the average vertical profile of the recorded images within a 2-mm-wide strip around $x_{\mathrm{fb}}$ [Fig. 2(a)]. The position of the profile's maximal vertical derivative, $z_{\mathrm{f}}$, provides a faithful measure of the height of the photo-accumulated front, which is easy to follow in time [Fig. 2(e)]. A heuristic description of its dynamics through the sigmoid function $z_{\mathrm{f}}(t)-z_{\mathrm{f}}(0)=$ $z_{\mathrm{f}}^{\infty} \exp \left[\left(t-t_{0}\right) / \delta\right] /\left\{\exp \left[\left(t-t_{0}\right) / \delta\right]+1\right\}$ can be used for both temporal registration, through a parameter to set a common origin of time $t_{0}$, and rescaling, by the characteristic falling time $\delta$. Typically, $\delta=15.5 \pm 6.6 \mathrm{~s}$ for $n_{0}^{\mathrm{h}}$, and
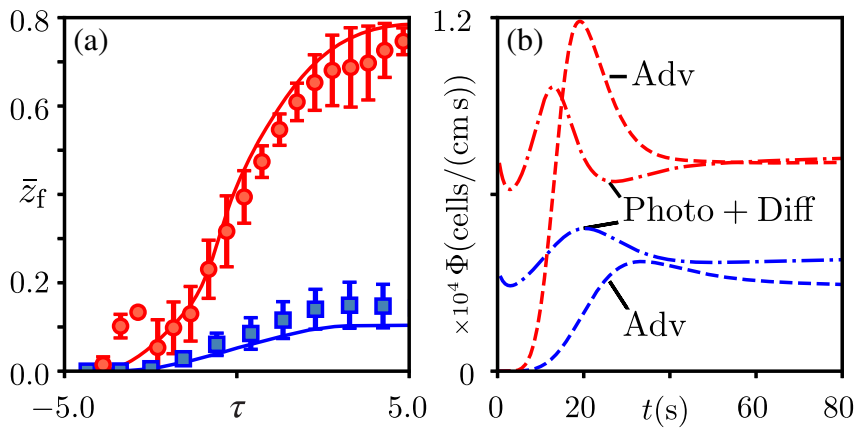

FIG. 3. Plume falling and flux balance. (a) Time evolution of the experimental (circles and squares) and numerical (solid lines) fronts vs $\tau$, for $z_{\mathrm{fb}} \sim 0.5 \mathrm{~cm}$. Simulations were run with $n_{0}=$ $1.5 \times 10^{7}$ cells $/ \mathrm{ml}$ (red line) and $n_{0}=7.0 \times 10^{6}$ cells $/ \mathrm{ml}$ (blue line); other parameters as described in the text. (b) Evolution of the advective (dashed lines) and phototactic plus diffusive (dot-dashed lines) fluxes a cross across a circular control surface of radius $0.18 \mathrm{~cm}$ centered on the optical fiber for the two cases described in panel (a). Same colour code.
$2.4 \pm 1.1 \mathrm{~s}$ for $n_{0}^{1}$ (errors are standard deviations of measurement sets). Figure 3(a) shows the average rescaled front dynamics $\bar{z}_{\mathrm{f}}(\tau)=\left[z_{\mathrm{f}}(\tau)-z_{\mathrm{f}}(0)\right] / z_{\mathrm{f}}(0)$ in terms of the intrinsic time $\tau=\left(t-t_{0}\right) / \delta$. The front falls almost to the bottom of the sample $\left(\bar{z}_{\mathrm{f}}=1\right)$ in the high concentration case $\left(n_{0}^{\mathrm{h}}\right.$ red circles), while in the low concentration case ( $n_{0}^{1}$ blue squares) the steady-state position is just $\sim 1 \mathrm{~mm}$ below the fiber $\left(\bar{z}_{\mathrm{f}} \simeq 0.1\right)$. This hints at the existence of a bifurcation between $n_{0}^{1}$ to $n_{0}^{\mathrm{h}}$.

The system's behavior, and the bifurcation, can be rationalized through a simple continuum model of $2 \mathrm{D}$ photo-bioconvection. The model describes the coupling between the local cell density, $n(\mathbf{x}, t)$, and the fluid flow, $\mathbf{u}(\mathbf{x}, t)[\mathbf{x}=(x, z)]$. The former obeys a continuity equation that includes contributions from the cells' active diffusion, phototaxis, and advection by the local background flow. The latter follows the Navier-Stokes equations, coupled to $n(\mathbf{x}, t)$ through the cells' excess density $(\Delta \rho)$ over the surrounding fluid (density $\rho_{\mathrm{F}}$ ). Following previous work $[57,58]$ this is captured in the Bousinnesq approximation. This minimal model recapitulates well the emergence and falling dynamics of a plume, and the geometric structure of the ensuing recirculation. Therefore, in keeping with a minimal-model approach, we will not consider gravitaxis [57], gyrotaxis [60], and the effect of cells' activity in both the bulk stress and the cell diffusivity tensors $[58,60]$, despite their role in phenomena like spontaneous bioconvection [58-60] and cells' focusing [46]. We note, however, that they could still contribute to a global rescaling of the dynamics. The system, contained within a square cavity of side $L$, is described by the following set of equations:

$$
\begin{aligned}
\nabla \cdot \mathbf{u} & =0, \\
\frac{\partial \mathbf{u}}{\partial t}+\mathbf{u} \cdot \nabla \mathbf{u} & =-\nabla p-n \hat{\mathbf{z}}+\sqrt{\frac{\mathrm{Sc}}{\mathrm{Ra}}} \nabla^{2} \mathbf{u}, \\
\frac{\partial n}{\partial t}+\nabla \cdot\left[\left(\mathbf{u}+\mathbf{u}_{\mathrm{ph}}\right) n\right] & =\frac{1}{\sqrt{\mathrm{RaSc}}} \nabla^{2} n,
\end{aligned}
$$

with no slip at the boundary, and no cell flux through the boundary. Despite using the same symbols for convenience, Eqs. (1)-(3) have been nondimensionalized by using $L$ as the characteristic length, and introducing the characteristic velocity for buoyancy-driven flow, $v_{\mathrm{c}}=$ $\left(n_{0} g \Delta \rho V_{\mathrm{CR}} L / \rho_{\mathrm{F}}\right)^{1 / 2}$, where $V_{\mathrm{CR}}=4 \pi r_{\mathrm{CR}}^{3} / 3$ is the estimated volume of an individual cell assuming a sphere of radius $r_{\mathrm{CR}}$. The characteristic time is then $t_{\mathrm{c}}=L / v_{\mathrm{c}}$, the scale for the (2D) pressure $p$ is given by $h \rho_{\mathrm{F}} v_{\mathrm{c}}^{2}$, and $n_{0}$ rescales the cell density. The behavior of the system is dictated by three nondimensional numbers: a Rayleigh number, $\mathrm{Ra}=\left(v_{\mathrm{c}} L\right)^{2} /(\nu D)$, and a Schmidt number, $\mathrm{Sc}=\nu / D$, based on the kinematic viscosity of the fluid $(\nu)$ and the cells' effective diffusivity $(D)$; and the phototactic sensitivity $\beta$, which governs the nondimensional phototactic term, $\mathbf{u}_{\mathrm{ph}}=\beta\left(v_{\mathrm{s}} / v_{\mathrm{c}}\right)\left(\left|\mathbf{x}-\mathbf{x}_{\mathrm{fb}}\right| / h^{*}\right) \nabla \boldsymbol{I} /|\nabla \boldsymbol{I}|_{\max }$. 
The phototactic drift, derived and tested in [31], includes the cells' swimming speed, $v_{\mathrm{s}}=7.8 \times 10^{-3} \mathrm{~cm} / \mathrm{s}$, and the effective thickness of the illuminated chamber, $h^{*}=$ $5.19 \times 10^{-2} \mathrm{~cm}$. The experimental system is expected to correspond to $\beta=0.14$ [31]. Other parameters are fixed to $\Delta \rho=0.05 \mathrm{~g} / \mathrm{cm}^{3}, \quad \rho_{\mathrm{F}}=1 \mathrm{~g} / \mathrm{cm}^{3}, \quad \nu=10^{-2} \mathrm{~cm}^{2} / \mathrm{s}$, $r_{\mathrm{CR}}=5 \times 10^{-4} \mathrm{~cm}$, and $D=3.9 \times 10^{-4} \mathrm{~cm}^{2} / \mathrm{s}[31,52]$. Cells are initially uniformly distributed within the quiescent fluid, and the vorticity-stream function formulations of Eqs. (1)-(3) are integrated with a spatially centered, second-order accurate, finite-difference scheme [61]; at each intermediate stage of the third-order Runge-Kutta method used to advance time, the Laplace equation for the stream function is solved with the conjugate gradient method [62]. The integration scheme was validated with benchmark solutions [63].

Figure 2 compares experimental and numerical dynamics of plume formation and sinking, as a function of the reduced time $\tau\left(z_{\mathrm{fb}}=0.5 \mathrm{~cm} ; n_{0}=n_{0}^{\mathrm{h}}\right)$. The agreement is excellent with no fitting parameters, and it is maintained also at longer times. Figure 2(d) shows the relative error between cells' stationary velocity field $\left[\mathbf{u}_{\mathrm{CR}}=\left(\mathbf{u}+\mathbf{u}_{\mathrm{ph}}\right) v_{\mathrm{c}}\right]$ from experiments and model, rescaled by their peak velocity. The small discrepancy ( $<25 \%$ on average) shows that the model captured well the structure of the photobioconvective flow of cells (see also Fig. 1). A closeup, in Fig. 3, on the experimental (circles and squares) and numerical (solid lines) front dynamics, proves that the model captures both sinking and nonsinking regimes (respectively $n_{0}^{\mathrm{h}}$ and $n_{0}^{1}$ ). We therefore decided to explore systematically the system's behavior in silico through a parametric sweep in the range $n_{0} \in[0.5,1.5] \times 10^{7}$ cells $/ \mathrm{ml}$ and $\beta \in[0.1,0.3]$. Figure 4 (a) shows that the steady-state front position $\left(z_{\mathrm{f}}^{\infty}\right)$ indicates the presence of two distinct

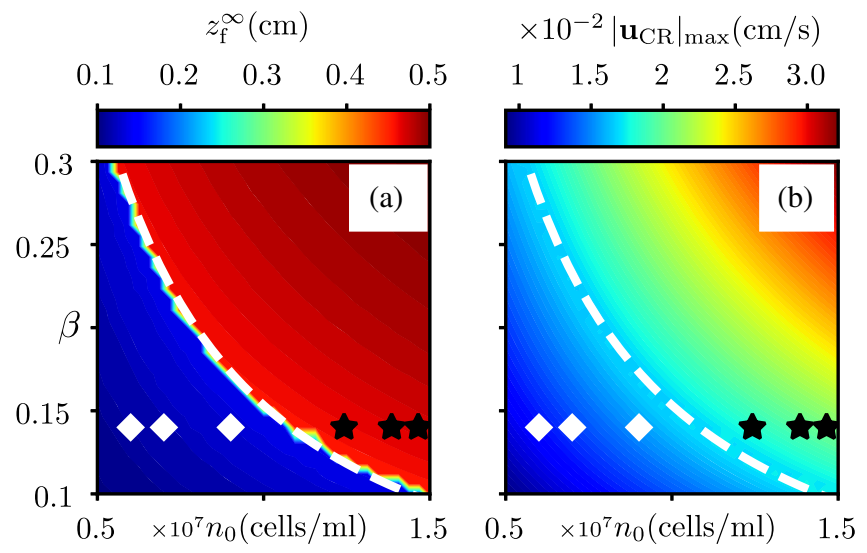

FIG. 4. Photobioconvective phase behavior. (a) Final position of the plume front, $z_{\mathrm{f}}^{\infty}$ and (b) maximum velocity of the cells, $\left|\mathbf{u}_{\mathrm{CR}}\right|_{\text {max }}$, vs initial cell density $n_{0}$ and photoadaptation parameter $\beta$. Plume forming (nonforming) experiments are indicated by black stars (white diamonds). The isoline $\left|\mathbf{u}_{\mathrm{CR}}\right|_{\max }=1.66 \times 10^{-2} \mathrm{~cm} / \mathrm{s}$ separates not falling and falling regimes (white dashed line). regimes separated by a sharp transition, in line with experiments (Fig. 4, diamond and star marks). There is a low- $n_{0}$ and low- $\beta$ regime, where cells accumulate but do not fall; and a high- $n_{0}$ and high- $\beta$ one, where the light induces a single, isolated bioconvective plume driving a vigorous global recirculation (Fig. 1). The two regimes are separated by a critical curve $\beta n_{0} \approx$ constant, corresponding to the isoline of maximum cells' velocity, $\left|\mathbf{u}_{\mathrm{CR}}\right|_{\max } \simeq 1.66 \times 10^{-2} \mathrm{~cm} / \mathrm{s}$. This is compatible with the full set of $n_{0}$ values explored experimentally [Fig. 4(b)]. The process driving the bifurcation can be understood intuitively by examining the balance between phototactic, diffusive, and advective fluxes of cells. Figure 3(b) shows the evolution of these fluxes across a circle of radius $1.8 \mathrm{~mm}$ centered on the optical fiber. Before the bifurcation (blue) advection (dot-dashed line) is always lower than the net flux due to cell motion (phototaxis and diffusion, dashed line): the front remains close to its initial position. Beyond the bifurcation (red) the downward advective flux dominates shortly after the initial accumulation, transporting cells downwards with a critical velocity arising from flux balance.

The ability to determine location and timing of the plume formation can be harnessed to govern the global transport properties of the suspension, e.g., to accelerate the active biomixing of nutrients. A simple procedure takes advantage of the left-right asymmetric flows generated when the light source is shifted from the midpoint of the chamber, as shown in the streamlines of Fig. 5(a) for a $\pm 1.5 \mathrm{~mm}$ shift
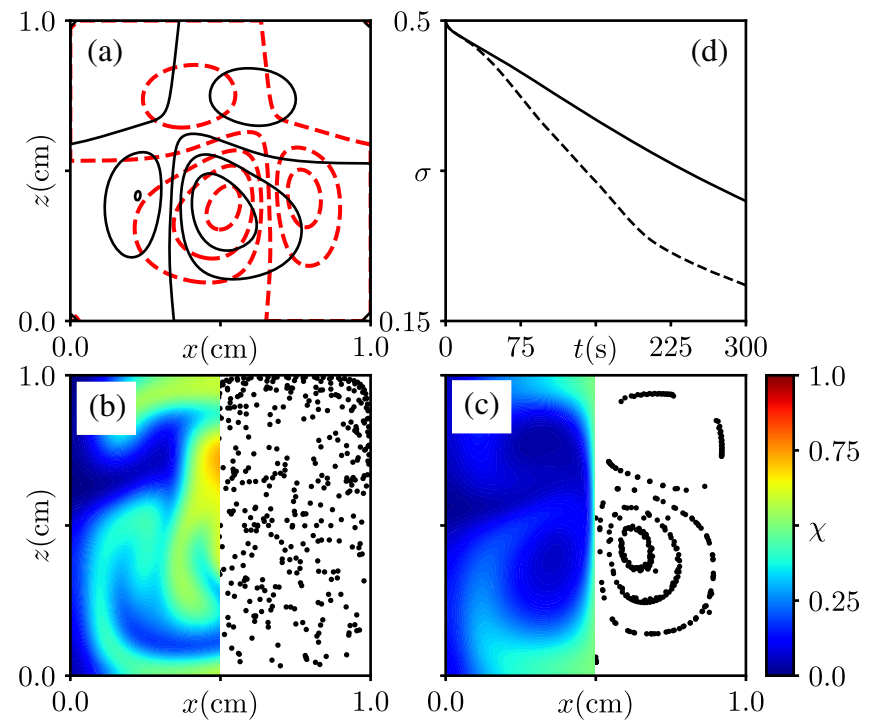

FIG. 5. Mixing by blinking plumes. (a) Streamlines of the induced flow at half a period (black line) and a full period (red line) of the time-dependent light protocol with $T \simeq 129 \mathrm{~s}$. (b), (c) Spatial distribution of $\chi$ (left-hand side) at $t \simeq 200 \mathrm{~s}$ and Poincaré map (right-hand side) after $i=75$ periods for the "blinking plumes" and the centered stationary case, respectively. (d) Time evolution of the spatial standard deviation of $\chi$ computed in (b) (dashed line) and (c) (solid line). 
(dashed red and solid black lines, respectively), and experimentally in the Supplemental Material, Movie S3 [51]. Alternating evenly between the two plumes in a cycle of period $T$, generates flow fields that display the characteristic crossing of streamlines required for efficient mixing in $2 \mathrm{D}$, and realize within a photobioconvective context the blinking vortex, a paradigmatic example of mixing by chaotic advection [64]. Figures 5(b) and 5(c) and the Supplemental Material, Movie S4 [51], show how the concentration $\chi$ of an advected nutrient of diffusivity $D_{\chi}=3 \times 10^{-3} \mathrm{~mm}^{2} / \mathrm{s}$, mimicking photosynthetically important gases like $\mathrm{CO}_{2}$ [65], evolves from an initial distribution localized in the right half of the container, for $t \sim 200 \mathrm{~s}$. Crossing of streamlines leads to the stretching and folding of thin filaments characteristic of chaotic advection. These in turn cause a significantly faster mixing than for a single steady plume [Fig. 5(c)], as seen in the decay of the standard deviation of the spatial concentration profile, $\sigma^{2}=$ $\left\langle(\chi-\langle\chi\rangle)^{2}\right\rangle$ [Fig. 5(d)] [66]. The origin of the enhanced mixing is evident in the Poincare maps obtained from the trajectories of ten tracer particles initially distributed uniformly along $z=5 \mathrm{~mm}$ and followed over $75 T$ [Figs. 5(b) and 5(c), right panels). Closed quasiperiodic orbits are readily visible for a stationary centered single plume while the "blinking plumes" lead to particles exploring most of the spatial domain. Active light patterning can therefore induce mixing advective maps, leading to strongly enhanced nutrient transport throughout the cell culture.

We have presented a novel mechanism that harnesses phototaxis to actively control a suspension of swimming microorganisms through their accumulation around a localized light source. The ensuing global instability, characterized by steady vortical flows for all parameter values, can easily lead to the emergence of isolated bioconvective plumes whose spatiotemporal localization is simply tuned by the external illumination. These properties contrast with the limited control afforded by standard bioconvection [41,67-70], and enable rapid light-mediated control of the flow, which can be used to regulate the transport properties of the suspension. The simple minimal model we use provides a surprisingly accurate quantitative description of the experimental system with no fitting parameters, but only when viewed in terms of the reduced time $\tau$. In terms of real time, plumes fall slower in experiments than in simulations: $\delta=10.5 \pm 4.7 \mathrm{~s}$ across all experiments beyond the bifurcation, compared to a range 4.5-4.8 s expected from the model. Interestingly, the quantitative agreement is much improved below the bifurcation (exp: $\delta=4.8 \pm 2.5 \mathrm{~s}$; num: $3.1-3.7 \mathrm{~s}$ ). The discrepancy beyond the bifurcation is possibly coming from a combination of disregarded swimming features (gravitaxis and gyrotaxis [57,60]) and confinement [71], to be disentangled in a future, dedicated study. Overall, together with recent work pioneering the use of radial stresses [45], our results set the stage to use light for fast and complex spatiotemporal control of the macroscopic dynamics of phototactic suspensions.

We acknowledge the support of the Ministerio de Ciencia, Innovación y Universidades (AEI, FEDER EU) Grants No. FIS2016-77692-C2-1-P (I. T.) and No. CTM-201783774-D (J. A.), and the subprogram Juan de la Cierva No. IJCI-2015-26955 (J. A.). J. A. is extremely grateful to Sara Guerrero for her enormous encouragement and support in the development of this work. M. P. and I. T. would like to thank Raymond Goldstein for support in the initial stages of the project. J.A. is grateful to Raphaël Jeanneret for thoughtful discussions.

* Corresponding author.

jarrieta@imedea.uib-csic.es

Corresponding author.

m.polin@warwick.ac.uk

Corresponding author.

ituval@imedea.uib-csic.es

[1] C. Chen, S. Liu, X. Q. Shi, H. Chaté, and Y. Wu, Nature (London) 542, 210 (2017).

[2] G. Ariel, A. Shklarsh, O. Kalisman, C. Ingham, and E. Ben-Jacob, New J. Phys. 15, 125019 (2013).

[3] J. E. Berleman and J. R. Kirby, FEMS Microbiol. Rev. 33, 942 (2009).

[4] R. L. Chisholm and R. A. Firtel, Nat. Rev. Mol. Cell Biol. 5, 531 (2004).

[5] P. Deng, L. De Vargas Roditi, D. Van Ditmarsch, and J. B. Xavier, New J. Phys. 16, 015006 (2014).

[6] M. C. Marchetti, J. F. Joanny, S. Ramaswamy, T. B. Liverpool, J. Prost, M. Rao, and R. A. Simha, Rev. Mod. Phys. 85, 1143 (2013).

[7] N. Koumakis, A. Lepore, C. Maggi, and R. Di Leonardo, Nat. Commun. 4, 2588 (2013).

[8] L. Angelani, C. Maggi, M. L. Bernardini, A. Rizzo, and R. Di Leonardo, Phys. Rev. Lett. 107, 138302 (2011).

[9] Z. Ma, Q. L. Lei, and R. Ni, Soft Matter 13, 8940 (2017).

[10] F. Kümmel, P. Shabestari, C. Lozano, G. Volpe, and C. Bechinger, Soft Matter 11, 6187 (2015).

[11] C. Bechinger, R. Di Leonardo, H. Löwen, C. Reichhardt, G. Volpe, and G. Volpe, Rev. Mod. Phys. 88, 045006 (2016).

[12] A. Aubret, M. Youssef, S. Sacanna, and J. Palacci, Nat. Phys. 14, 1114 (2018).

[13] P. Denissenko, V. Kantsler, D. J. Smith, and J. KirkmanBrown, Proc. Natl. Acad. Sci. U.S.A. 109, 8007 (2012).

[14] H. Wioland, E. Lushi, and R. E. Goldstein, New J. Phys. 18, 075002 (2016).

[15] S. Thutupalli, D. Geyer, R. Singh, R. Adhikari, and H. A. Stone, Proc. Natl. Acad. Sci. U.S.A. 115, 5403 (2018).

[16] L. Giomi, New J. Phys. 18, 081001 (2016).

[17] A. Morin and D. Bartolo, Phys. Rev. X 8, 021037 (2018).

[18] P. Galajda, J. Keymer, P. M. Chaikin, and R. Austin, J. Bacteriol. 189, 8704 (2007).

[19] V. Kantsler, J. Dunkel, M. Polin, and R. E. Goldstein, Proc. Natl. Acad. Sci. U.S.A. 110, 1187 (2013). 
[20] T. Ostapenko, F. J. Schwarzendahl, T. J. Böddeker, C. T. Kreis, J. Cammann, M. G. Mazza, and O. Bäumchen, Phys. Rev. Lett. 120, 068002 (2018).

[21] E. Lushi, H. Wioland, and R. E. Goldstein, Proc. Natl. Acad. Sci. U.S.A. 111, 9733 (2014).

[22] A. Bricard, J. B. Caussin, N. Desreumaux, O. Dauchot, and D. Bartolo, Nature (London) 503, 95 (2013).

[23] G. Vizsnyiczai, G. Frangipane, C. Maggi, F. Saglimbeni, S. Bianchi, and R. Di Leonardo, Nat. Commun. 8, 15974 (2017),

[24] S.-H. Lee, Y. Roichman, and D. G. Grier, Opt. Express 18, 6988 (2010).

[25] D. Stellinga, M. E. Pietrzyk, J. M. Glackin, Y. Wang, A. K. Bansal, G. A. Turnbull, K. Dholakia, I. D. Samuel, and T. F. Krauss, ACS Nano 12, 2389 (2018).

[26] A. N. Dodd, J. Kusakina, A. Hall, P. D. Gould, and M. Hanaoka, Photosynth. Res. 119, 181 (2014).

[27] Z. Li, S. Wakao, B. B. Fischer, and K. K. Niyogi, Annu. Rev. Plant Biol. 60, 239 (2009).

[28] D. P. Häder, Microbiol. Rev. 51, 1 (1987).

[29] D.-P. Häder and M. Lebert, in Chemotaxis: Methods and Protocols, Methods in Molecular Biology Vol. 571, edited by T. Jin and D. Hereld (Humana Press, Totowa, 2009), Chap. 3, pp. 51-65.

[30] K. Drescher, R. E. Goldstein, and I. Tuval, Proc. Natl. Acad. Sci. U.S.A. 107, 11171 (2010).

[31] J. Arrieta, A. Barreira, M. Chioccioli, M. Polin, and I. Tuval, Sci. Rep. 7, 3447 (2017).

[32] A. Giometto, F. Altermatt, A. Maritan, R. Stocker, and A. Rinaldo, Proc. Natl. Acad. Sci. U.S.A. 112, 7045 (2015).

[33] K. C. Leptos, M. Chioccioli, S. Furlan, A. I. Pesci, and R. E. Goldstein, https://dx.doi.org/10.1101/254714.

[34] J. Arlt, V. A. Martinez, A. Dawson, T. Pilizota, and W. C. Poon, Nat. Commun. 9, 768 (2018).

[35] X. Jin and I. H. Riedel-Kruse, Proc. Natl. Acad. Sci. U.S.A. 115, 3698 (2018).

[36] Y. Huang, A. Xia, G. Yang, and F. Jin, ACS Synth. Biol. 7, 1195 (2018).

[37] C. Maggi, F. Saglimbeni, M. Dipalo, F. De Angelis, and R. Di Leonardo, Nat. Commun. 6, 7855 (2015).

[38] C. Lozano, B. ten Hagen, H. Löwen, and C. Bechinger, Nat. Commun. 7, 12828 (2016).

[39] B. Dai, J. Wang, Z. Xiong, X. Zhan, W. Dai, C.-C. Li, S.-P. Feng, and J. Tang, Nat. Nanotechnol. 11, 1087 (2016).

[40] K. Ozasa, J. Lee, S. Song, M. Hara, and M. Maeda, Lab Chip 11, 1933 (2011).

[41] E. Shoji, H. Nishimori, A. Awazu, S. Izumi, and M. Iima, J. Phys. Soc. Jpn. 83, 043001 (2014).

[42] A. T. Lam, K. G. Samuel-Gama, J. Griffin, M. Loeun, L. C. Gerber, Z. Hossain, N. J. Cira, S. A. Lee, and I. H. RiedelKruse, Lab Chip 17, 1442 (2017).

[43] A. C. Tsang, A. T. Lam, and I. H. Riedel-Kruse, Nat. Phys. 14, 1216 (2018).

[44] D. B. Weibel, P. Garstecki, D. Ryan, W. R. DiLuzio, M. Mayer, J. E. Seto, and G. M. Whitesides, Proc. Natl. Acad. Sci. U.S.A. 102, 11963 (2005).
[45] J. Dervaux, M. Capellazzi Resta, and P. Brunet, Nat. Phys. 13, 306 (2017).

[46] X. Garcia, S. Rafaï, and P. Peyla, Phys. Rev. Lett. 110, 138106 (2013).

[47] M. A. Borowitzka, J. Biotechnol. 70, 313 (1999).

[48] H. C. Greenwell, L. M. L. Laurens, R. J. Shields, R. W. Lovitt, and K. J. Flynn, J. R. Soc. Interface 7, 703 (2010).

[49] S. A. Scott, M. P. Davey, J. S. Dennis, I. Horst, C. J. Howe, D. J. Lea-Smith, and A. G. Smith, Curr. Opin. Biotechnol. 21, 277 (2010).

[50] E. Harris, The Chlamydomonas Sourcebook (Academic Press, Oxford, 2009), Vol. 2.

[51] See Supplemental Material at http://link.aps.org/ supplemental/10.1103/PhysRevLett.123.158101 for measured intensity profile from the optical fiber vs best Gaussian fit.

[52] M. Polin, I. Tuval, K. Drescher, J. P. Gollub, and R. E. Goldstein, Science 325, 487 (2009).

[53] K. W. Foster and R. D. Smyth, Microbiol. Rev. 44, 572 (1980).

[54] S. Kateriya, G. Nagel, E. Bamberg, and P. Hegemann, Physiology 19, 133 (2004).

[55] K. Josef, J. Saranak, and K. W. Foster, Cell Motil. Cytoskeleton 63, 758 (2006).

[56] Z. J. Taylor, R. Gurka, G. A. Kopp, and A. Liberzon, IEEE Trans. Instrum. Meas. 59, 3262 (2010).

[57] S. Childress, M. Levandowsky, and E. A. Spiegel, J. Fluid Mech. 69, 591 (1975).

[58] T. J. Pedley and J. O. Kessler, Annu. Rev. Fluid Mech. 24, 313 (1992).

[59] M. A. Bees and N. A. Hill, Phys. Fluids 10, 1864 (1998).

[60] T. J. Pedley and J. O. Kessler, J. Fluid Mech. 212, 155 (1990).

[61] D. Anderson, J. Tannehill, and R. Pletcher, Computational Fluid Mechanics and Heat Transfer, Series in Computational Methods in Mechanics and Thermal Sciences (Hemisphere Publishing Corporation, Washington DC, 1984).

[62] J. Ferziger and M. Peric, Computational Methods for Fluid Dynamics (Springer, Berlin, Heidelberg, 2001).

[63] G. De Vahl Davis and I. P. Jones, Int. J. Numer. Methods Fluids 3, 227 (1983).

[64] H. Aref et al., Rev. Mod. Phys. 89, 025007 (2017).

[65] A. A. F. Mazarei and O.C. O. Sandall, AIChE J. 26, 154 (1980).

[66] A. D. Stroock, S. K. W. Dertinger, A. Ajdari, I. Mezic, H. A. Stone, and G. M. Whitesides, Science 295, 647 (2002).

[67] R. V. Vincent and N. A. Hill, J. Fluid Mech. 327, 343 (1996).

[68] C. R. Williams and M. A. Bees, J. Fluid Mech. 678, 41 (2011).

[69] C. R. Williams and M. A. Bees, J. Exp. Biol. 214, 2398 (2011).

[70] M. K. Panda and R. Singh, Phys. Fluids 28, 054105 (2016).

[71] M. Pushkin and M. A. Bees, in Biophysics of Infection, Advances in Experimental Medicine and Biology, edited by M. C. Leake (Springer, New York, 2016), Chap. 12, pp. 193-205. 